\title{
Absorbing Inlet Boundary Analysis of Rotor Wake/Stator Time Domain Computations
}

\author{
Martin Olausson* and Lars-Erik Eriksson ${ }^{\dagger}$ \\ Chalmers University of Technology, SE-412 96 Gothenburg, Sweden
}

In this paper, different solvers and methods of how to apply absorbing boundary condition at the inlet of nonlinear rotor wake/stator computations are analyzed. Absorbing boundaries are used in order to avoid spurious reflections of acoustic waves at the inlet and outlet of the computational domain. A URANS solver with chorochronic, time lag, periodic boundaries is used to solve the unsteady flow inside a stator vane passage. Boundary conditions of 1D type (axial characteristics) and 2D type (axial-tangential characteristics) are applied at the inlet to the stator domain. Another method, using a rotating inlet buffer zone, is also analyzed. The wake is stationary in this rotating inlet zone and a standard buffer layer method can be used in the rotating frame of reference without destroying the wake. A solver based on the harmonic balance technique is also tested and compared to the standard time integration method. These boundary conditions and solvers are analyzed for a realistic high bypass ratio stator vane geometry, considering tone noise generation from the impingement of rotor wakes on the stators.

\section{Nomenclature}

Roman

$k \quad$ stator BPF index, wave number

$L \quad$ length

$m \quad$ tangential mode number

$n \quad$ rotor BPF index

$N \quad$ number of blades/time levels/blade passing frequencies

$p \quad$ pressure

$q \quad$ state vector in flow equations in primitive variable form in cylindrical coordinate system

$Q \quad$ state vector in flow equations in conservative form

S entropy

$t \quad$ time

$T \quad$ period, inverse of BPF

$u_{i} \quad$ components of velocity vector

Greek

$\epsilon \quad$ damping factor

$\theta \quad$ tangential coordinate

$\lambda \quad$ wave length

$\rho \quad$ density

$\phi \quad$ Fourier coefficients of state vector

$\omega \quad$ angular frequency $(\omega=2 \pi f)$

$\Omega \quad$ rotor shaft speed

Subscript

$r \quad$ radial component

*PhD student, Division of Fluid Dynamics, Department of Applied Mechanics, Chalmers University of Technology, SE-412 96 Göteborg, Sweden.

†Professor, Division of Fluid Dynamics, Department of Applied Mechanics, Chalmers University of Technology, SE-412 96 Göteborg, Sweden. 


\begin{tabular}{ll}
$x$ & axial component \\
$\theta$ & tangential component \\
Superscript & \\
\multicolumn{1}{c}{ Fourier representation } \\
$-\quad$ time average \\
Abbreviations \\
$B L \quad$ Buffer Layer \\
$B P F$ & Blade Passing Frequency \\
$C A A$ & Computational Aeroacoustics \\
$C F D$ & Computational Fluid Dynamics \\
LNSE & Linearized Navier-Stokes Equations \\
$M P I$ & Message Passing Interface \\
$T L$ & Time Level \\
URANS & Unsteady Reynolds-Averaged Navier-Stokes
\end{tabular}

\section{Introduction}

Computational aeroacoustics is a promising candidate for noise predictions of future engine configurations. In order to meet increasingly stricter regulations it is important to address noise at an early stage in the design process of new aircraft engines. This paper focuses on CAA computations of the rotor wake interaction with the stators in a fan stage, which is an important source of noise. The stator domain is discretized, and the rotor wakes are specified at the inflow to the domain. Reflections of acoustic waves at both the inlet and outlet of the computational domain must be minimized to avoid contamination. There are mainly two approaches for avoiding reflections that can be used separately or combined: (1) absorbing boundary conditions, i.e. the boundary condition is suited to minimize reflections, ${ }^{1-3}$ and (2) buffer layer, i.e. a region near the boundary that is treated to artificially absorb incoming waves. ${ }^{4} \mathrm{~A}$ standard buffer layer that damps all unsteadiness in the current solution can be used at the outlet of the domain. At the inlet, on the other hand, the rotor wakes must be preserved. A standard buffer layer can not be applied in a frame of reference where the rotor wake is not stationary. One alternative is to try to find a reference solution close to the inlet that contains the downstream traveling wake. The upstream traveling pressure waves can then be damped out by a buffer layer that uses the reference solution as a target solution. The reference solution can be obtained from a rotor simulation solved in a rotating frame of reference. A three dimensional section of the outlet to the rotor computational domain must then be interpolated to the inlet section of the stator computational domain. This must be done at each time step since the reference solution must follow the rotating rotor wake in the stator domain. To avoid 3D interpolation, another alternative is to let a part of the inlet rotate with the wake at the same speed as the rotor. The wake is stationary in this rotating inlet zone, and a standard buffer layer method can be used there without destroying the wake. ${ }^{5}$ The rotating inlet buffer layer should have the same periodicity as the rotor and a chorochronic, Fourier-based in time and tangential direction, blade row interface can then be used between the rotating domain and the stator domain.

\section{Flow Solver}

The governing equations for flow induced noise generation are the Navier-Stokes equations. The solver, based on the G3D family of codes, solves the nonlinear unsteady, Favre-filtered, Reynolds-averaged NavierStokes (URANS) equations in conservative form with a realizable k-epsilon turbulence model. It is assumed that there is a scale separation between the predicted unsteady effects and turbulent fluctuations, and a limit on the length scale in the turbulence model is therefore introduced. The discretization of the domain is done with a boundary-fitted, curvilinear, non-orthogonal multi-block mesh. The fluxes are reconstructed with a standard third-order upwind scheme for the convective part and a second-order centered difference scheme is used for the diffusive part. The solution is updated with a three-stage Runge-Kutta technique. The solver is parallelized using MPI libraries to enable multi-processor computations. ${ }^{6-9}$ 


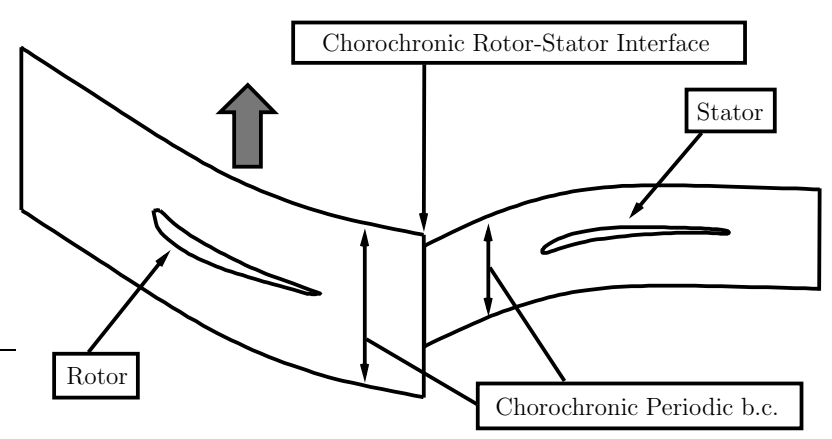

Figure 1. Schematic of Rotor and Stator Computational Domain

\section{Chorochronic Periodicity}

A rotor-stator interaction computation can be simplified in terms of computational mesh size by using chorochronic boundary conditions and interface. ${ }^{9-16}$ Only one blade per blade row needs to be discretized, and the chorochronic periodic boundary conditions, i.e. periodic with time lag, ensure that the Tyler-Sofrin interaction modes, $m$ in Eqn. (1), fit both the rotor and stator computational domains. ${ }^{17}$

$$
m_{n, k}=n N_{\text {rotor }}+k N_{\text {stator }}
$$

The rotor domain, solved in a rotating frame of reference, is connected to the stator domain by a chorochronic, Fourier based, rotor-stator interface. The Tyler-Sofrin tangential modes are calculated on both sides of the interface for each radial position and are transferred to the other frame of reference by a shift in frequency; these modes contain all the deterministic unsteadiness of the flow. ${ }^{9-11}$ A schematic of the computational set-up for a rotor-stator interaction computation using chorochronic boundary conditions and interface is shown in Fig. 1.

Time Fourier coefficients of the flow variables are calculated and continuously updated close to the chorochronic boundaries with a moving average technique. ${ }^{9,10}$ It can be derived from the definition of how a Fourier coefficient is calculated by integration over one period. In the stator blade row, that is:

$$
\phi_{n}=\frac{1}{T} \int_{t-T}^{t} Q\left(t^{\prime}\right) e^{-i \omega_{n} t^{\prime}} d t^{\prime}
$$

where $\phi_{n}$ is the $n^{t h}$ harmonic Fourier coefficients to the state vector, $Q$. The fundamental period, $T$, in the stator frame of reference is the inverse of the rotor $\mathrm{BPF}$ and is calculated from the difference in the angular velocity between the two blade rows and the number of rotor blades as:

$$
T_{\text {rotor }}=\frac{2 \pi}{N_{\text {rotor }}\left|\Omega_{\text {rotor }}-\Omega_{\text {stator }}\right|}
$$

The angular frequency, $\omega_{n}$, of each harmonic is calculated as:

$$
\omega_{n}=n N_{\text {rotor }}\left(\Omega_{\text {rotor }}-\Omega_{\text {stator }}\right)
$$

The time derivatives to the Fourier coefficients can be calculated from the integration boundaries of Eqn. $(2)$,

$$
\frac{d \phi_{n}}{d t}=\frac{1}{T}\left[Q(t) e^{-i \omega_{n} t}-Q(t-T) e^{-i \omega_{n}(t-T)}\right]
$$

where the state of the flow one period back in time can be approximated by evaluating the Fourier series as:

$$
Q(t-T) \approx \widehat{Q}(t)=\sum_{n} \phi_{n} e^{i \omega_{n} t}
$$

This can be used to find an approximate value of the Fourier coefficient derivative:

$$
\frac{d \phi_{n}}{d t}=\frac{1}{T}\left[Q(t)-\sum_{l} \phi_{l} e^{i \omega_{l} t}\right] e^{-i \omega_{n} t}
$$

$$
3 \text { of } 15
$$

American Institute of Aeronautics and Astronautics 
This is done in the area close to the periodic boundary to acquire a Fourier representation of the flow there. The coefficients are evaluated at a different time to obtain the time lagged state that corresponds to the state on the other side of the periodic boundary.

Some extra numerical damping is needed to stabilize the periodic boundary with a time lag. ${ }^{9,11-15}$ The method used in this paper is that the Fourier coefficients are employed to damp out nonperiodic flow phenomena close to the periodic boundaries. The Fourier coefficients are then evaluated again, at the current time, and used in the cell where the sampling occurred and are compared to the state there in order to calculate a temporal damping term. The damping term is added to the state vector derivative as:

$$
\frac{d Q}{d t}=\cdots-\epsilon[Q(t)-\widehat{Q}(t)]
$$

where $\epsilon$ is the damping factor. This damping term will be zero when the computation has converged to a periodic solution. An important advantage of this method is that no extra spatial dissipation is added.

Time Fourier series representations of the flow in the area close to the rotor-stator interface are also updated according to Eqn. (7). This should be done for the primitive variables, $q$, in a cylindrical coordinate system.

$$
q=\left[\begin{array}{c}
\rho \\
u_{x} \\
u_{r} \\
u_{\theta} \\
p
\end{array}\right]
$$

Sampling is done on both sides of the interface to get a complete representation of the flow information in both time and space. The derivation is done for a constant radius section of the interface:

$$
\begin{aligned}
& \frac{d \phi_{n}^{\text {stator }}(\theta)}{d t}=\frac{1}{T_{\text {rotor }}}\left[q(t, \theta)-\sum_{l} \phi_{l}^{\text {stator }}(\theta) e^{i \omega_{l} t}\right] e^{-i \omega_{n} t} \\
& \frac{d \phi_{k}^{\text {rotor }}(\theta)}{d t}=\frac{1}{T_{\text {stator }}}\left[q(t, \theta)-\sum_{l} \phi_{l}^{\text {rotor }}(\theta) e^{i \omega_{l} t}\right] e^{-i \omega_{k} t}
\end{aligned}
$$

where the angular frequencies and the fundamental period of the modes in the rotor frame of reference are calculated by Eqn. (12-13), i.e. the frequency depends only on the stator harmonics, $k$, and the stator BPF.

$$
\begin{gathered}
\omega_{k}=k N_{\text {stator }}\left(\Omega_{\text {stator }}-\Omega_{\text {rotor }}\right) \\
T_{\text {stator }}=\frac{2 \pi}{N_{\text {stator }}\left|\Omega_{\text {stator }}-\Omega_{\text {rotor }}\right|}
\end{gathered}
$$

The time and space Fourier coefficients of the modes, $m_{n, k}$, are calculated by integrating the time Fourier coefficients over each sector in the tangential direction.

$$
\begin{gathered}
\phi_{n, k}=\frac{N_{\text {stator }}}{2 \pi} \int_{0}^{\frac{2 \pi}{N_{\text {stator }}}} \phi_{n}^{\text {stator }}(\theta) e^{i m_{n, k} \theta} d \theta \\
\phi_{n, k}=\frac{N_{\text {rotor }}}{2 \pi} \int_{0}^{\frac{2 \pi}{N_{\text {rotor }}}} \phi_{k}^{\text {rotor }}(\theta) e^{i m_{n, k} \theta} d \theta
\end{gathered}
$$

These modes can now be passed on to any rotating frame of reference by a shift in frequency. The frequency according to Eqn. (4) is used in the stator frame of reference, and the frequency according to Eqn. (12) in the rotor frame of reference. The evaluation is made by a summation over all the Fourier modes.

$$
\begin{aligned}
& \widehat{q}_{\text {stator }}(t, \theta)=\sum_{n} \sum_{k} \phi_{n, k} e^{i \omega_{n} t} e^{-i m_{n, k} \theta} \\
& \widehat{q}_{\text {rotor }}(t, \theta)=\sum_{n} \sum_{k} \phi_{n, k} e^{i \omega_{k} t} e^{-i m_{n, k} \theta}
\end{aligned}
$$




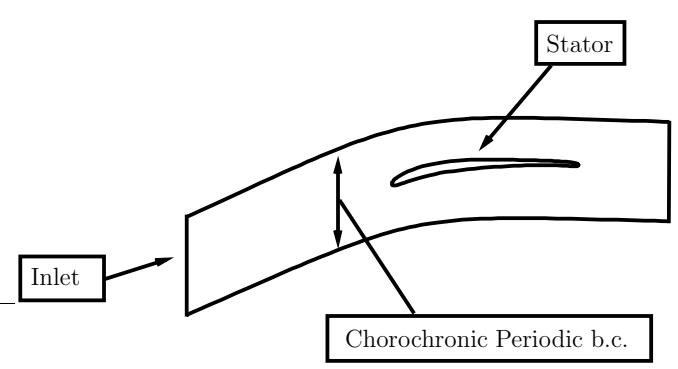

Figure 2. Schematic of Rotor Wake/Stator Computational Domain. The Rotor Wake in Specified at the Inlet.

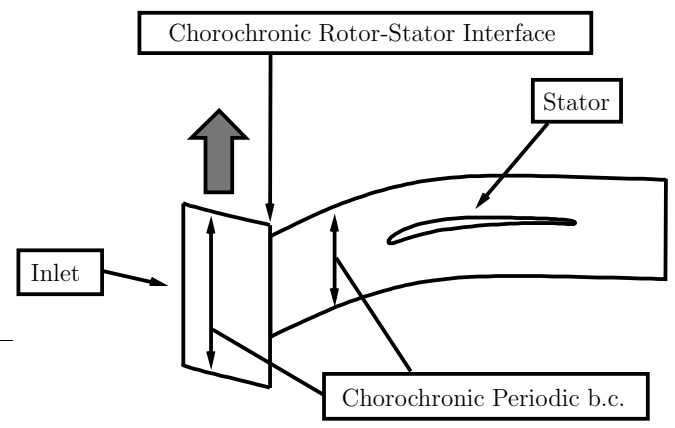

Figure 3. Schematic of Inlet Buffer Layer and Stator Computational Domain. The Rotor Wake is Specified at the Inlet.

Note that it is assumed in Eqn. (16-17) that both positive and negative frequencies are used.

$$
\begin{aligned}
& n=\ldots-1,0,1 \ldots \\
& k=\ldots-1,0,1 \ldots
\end{aligned}
$$

Some simplifications can be made by calculating coefficients only for positive frequencies, and then using the complex conjugates to obtain the full matrix of Fourier coefficients to the modes.

$$
\phi_{-m}=\phi_{-n,-k}=\phi_{n, k}^{*}=\phi_{m}^{*}
$$

\section{Absorbing Boundaries}

Many fluid flow phenomena, such as aeroacoustics, are unsteady, and waves may reflect spuriously at the inflow/outflow boundaries. The boundaries should be made absorbing to avoid contamination by these reflections.

The rotor is often omitted in rotor stator interaction computations to limit the size of the computational domain even more, and the rotor wake is then specified at the inlet to the stator domain, Fig. 2. Fully coupled rotor stator interaction computations are also very difficult with frequency domain linearized Navier-Stokes equations solvers, FD-LNSE, ${ }^{18}, 19$ since the solution is obtained by solving for each frequency separately, i.e. perturbations of harmonics to the blade passing frequency are solved on top of a mean flow solution separately. The modes with the same frequency in one frame of reference all have different frequencies in the other frame of reference.

Absorbing boundary conditions of the 1D type are commonly used in both steady and unsteady computations. ${ }^{1,2}$ They are robust and work well for waves normal to the boundary. The Tyler-Sofrin interaction waves in turbomachinery computations cannot all be normal to an inlet or outlet boundary inside a duct, and something more than a 1D absorbing boundary condition is needed. Absorbing boundaries of a 2D type (axial-tangential characteristics) have been used in both FD-LNSE solvers and nonlinear Euler equations solvers. ${ }^{3,18}$ The number of tangential modes that should be taken care of in the $2 \mathrm{D}$ absorbing boundary is limited for FD-LNSE solvers to the Tyler-Sofrin interaction modes of the frequency that is currently solved for. In the nonlinear case, on the other hand, all the modes for all frequencies of interest must be taken 
care of at the same time, since the solution for all frequencies is obtained in one simulation. This makes a 2D absorbing b.c. more complex for a nonlinear computation, and it is still not perfect for a 3D case. 3D absorbing b.c., built on the analysis by Verdon, ${ }^{19}$ can be used in FD-LNSE solvers, but it would in the author's opinion be a bit too complex to be of practical use in nonlinear computations. All tangential modes must then be split into radial modes as well, and the effort of finding them will dominate the computation.

Absorbing buffer layers can be used in a region close to a boundary to damp out unsteady waves. ${ }^{4}$ The buffer layer involves the use of a low pass filter and is easily implemented in a CFD solver. As discussed earlier it cannot be used if the boundary condition is unsteady, as for instance in an inlet to the stator domain with a specified wake profile, in a rotor wake/stator computation as shown in Fig. 2. However, if a part of the inlet rotates with the rotor wake, as shown in Fig. 3, a standard buffer layer can be used in the rotating frame of reference without destroying the wake. If the buffer layer is well tuned it will damp out both the tangential and radial modes of all frequencies.

The buffer layer is based on a low pass filter. A time average of the state vector in the buffer zone is compared to the current solution, and a damping term can be calculated and added to the state vector derivative,

$$
\frac{d Q}{d t}=\cdots-\epsilon[Q(t)-\bar{Q}(t)]
$$

The damping factor, $\epsilon$, can be a constant value or varied throughout the buffer layer. A small value in the beginning of the buffer layer and a larger value close to the boundary is usually good to avoid reflections in the buffer layer itself. ${ }^{4,20}$

\section{Harmonic Balance Technique}

The harmonic balance technique can be used for time periodic flows in turbomachines. ${ }^{21,22}$ The time periods of the flow are then enforced by the solver instead of iterating until a periodic solution is obtained as in a standard URANS method. The solver solves for a discrete number of time levels over the period when the harmonic balance technique is used, i.e. a set of flow variables are stored for each time level and each cell in the computational mesh. A spectral time derivative is calculated from a linear combination of the solutions at the different time levels and a pseudo time marching technique is used to iterate towards convergence. The time periods are different in the rotor and the stator domain and thus the time levels are not the same either, but the two domains can still be connected by a rotor-stator interface.

The same solver as described in section II is used to calculate all the fluxes but this is now done for each time level and the spectral time derivative is added as a source term. It is by the spectral time derivative that the time levels are connected. The Fourier coefficients in the Fourier based boundary conditions are calculated directly instead of by sampling, as described in section III, since the solution of an entire period is available at each iteration. Further on there is no need for any extra numerical damping in the periodic boundary with time lag since the solution is always periodic. Also, the time average of the state vector used in the buffer layers is simply the mean value of the time levels.

There is a simple relation between how many time levels that should be used and the number of harmonics that can be resolved,

$$
N_{T L}=2 N_{B P F}+1
$$

Calculations has shown however that it might not be sufficient to include only the frequencies that are of interest. Some additional higher frequencies must be included to avoid truncation errors at the frequencies of interest since the nonlinear effects allow for exchange of energy between them.

This method has the potential of being orders of magnitude faster than a standard time accurate method but convergence is not guaranteed, as will be discussed in section VI.

\section{Results and Discussion}

A stator vane of a realistic high bypass ratio fan stage with a rotor-stator blade count ratio of about 1:1.56 has been discretized, and all acoustic waves up to the 3rd BPF are resolved by at least 12 grid points per wavelength with the current resolution. The total number of cells for one stator passage is about 3.5M. The blade and hub surface mesh for every other node is shown in Fig. 4. The rotor wake is obtained from 


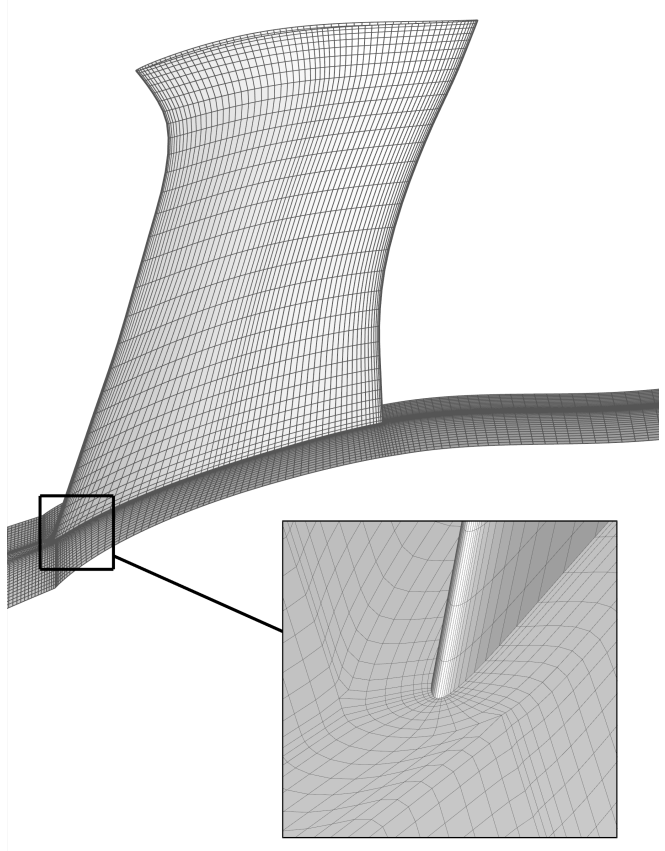

Figure 4. Blade and Hub Surface Mesh (Every Other Node Shown)

Table 1. Specification of Cases

\begin{tabular}{ll}
\hline Case I a & 1D absorbing inlet. \\
Case I b & 2D absorbing inlet. \\
Case II a & 1D absorbing inlet without inlet buffer layer. \\
Case II b & 1D absorbing inlet with inlet buffer layer. \\
Case II c & 1D absorbing inlet with inlet buffer layer solved \\
& with the harmonic balance technique. \\
\hline
\end{tabular}

a separate CFD computation in the rotor frame of reference, and the inlet to the stator domain is modified into two different cases.

\section{VI.A. Case I}

This computation is done entirely in the stator frame of reference, with the rotor wake specified at the inlet to the domain. Static pressure is specified at the outlet boundary and a region before the outlet is damped with a buffer layer. Periodic boundaries with time lag, chorochronic, are specified at the pitch-wise boundaries. Case I has been computed with inlet boundary conditions of $1 \mathrm{D}$ type (a) and 2D type (b). A modification in the $2 \mathrm{D}$ type boundary condition was necessary to make it stable. If an acoustic mode is cut off or close to cut off, according to 2D analysis, it is set to zero in the boundary condition. Case I (b) did not converge to a periodic solution without this modification. Figure 5 shows the blade and hub surface of case I.

\section{VI.B. Case II}

A part of the inlet section is extended in case II in the pitch-wise direction to fit the rotor pitch, as shown in Fig. 6. The inlet section is solved in a rotating frame of reference and a chorochronic rotor-stator interface connects the rotating inlet section with the stationary domain. The rotor wake is specified at the inlet, and a region between the inlet and the rotor-stator interface can be damped with a buffer layer. The wake is 


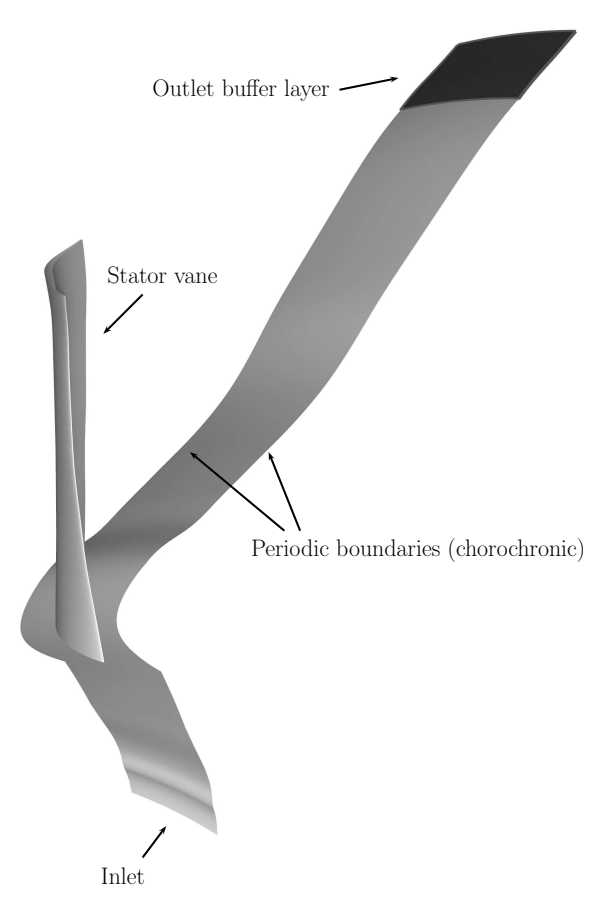

Figure 5. Case I, Blade and Hub Surface

stationary in the rotating frame of reference and is therefore not dissipated by the buffer layer. The axial position of the inlet and the wake specification is the same in both case I and case II, and the inlet boundary condition is of a 1D absorbing type. Case II has been computed both without (a) and with (b,c) an inlet buffer layer in the inlet zone. The outlet buffer layers in case I $(a, b)$ and case II (a) have the same constant value of the damping factor, $\epsilon$. In case II $(b, c)$ however, the damping factor was varied along the axial coordinate to follow an hyperbolic tangent curve both at the inlet and at the outlet. Case II (c) is exactly the same as case II (b) but solved with the harmonic balance technique. Thirteen time levels was used in the harmonic balance calculations, i.e. the blade passing frequency and five harmonics are resolved but only the frequencies up to the third BPF are of interest. Table 1 shows a summary of the different cases.

A case based on case I (a) has been calculated with the harmonic balance technique but this case did not converge. There was a slowly growing pressure mode close to the hub between the inlet and the stator that seemed to origin from the boundary layer. The frequency of the instability probably fits the stator domain frequencies since this did not appear in case II (c) where the the solver solves for other frequencies in the rotating inlet section.

\section{VI.C. Tangential mode analysis}

The solutions have been obtained by first solving the mean flow for both cases. The rotor wake is then added, the chorochronic boundary conditions are applied, and a converged periodic solution is obtained from a time accurate simulation of about 40 rotor blade passing periods for the URANS cases, i.e. all except case II (c) which has been run to convergence. The solution is sampled over one rotor blade passing period, and Fourier coefficients of the 1st, 2nd and 3rd BPF are calculated in the stator frame of reference together with the mean flow. The Tyler-Sofrin tangential mode Fourier coefficients are then calculated from the Fourier coefficients in time. This is done between the inlet and the stator vane at the same axial position in both cases, and lies between the rotor-stator interface and the stator vane in case II.

The mean flow is the same for all cases. Figures 7- 8 show the modes of the rotor harmonics of entropy and pressure fluctuations, respectively. Cases I (a) and II (a,b,c) correspond well to each other except for a small difference in entropy at the 3rd BPF. This implies that the wake specification is unaffected by the buffer layer and the rotor-stator interface in case II. It is expected that slightly more entropy should be generated, especially for the higher frequencies, in case I since the wake rotates a longer distance in the 


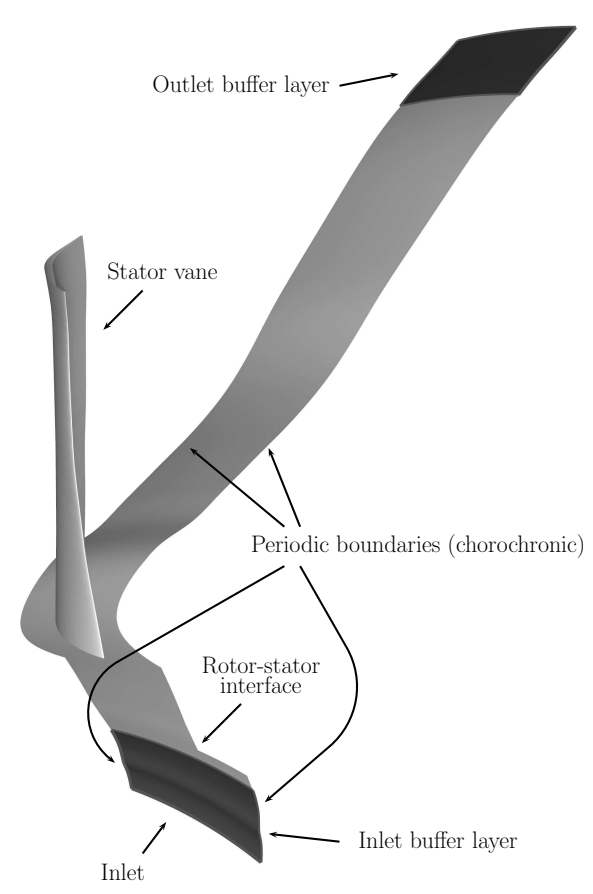

Figure 6. Case II, Blade and Hub Surface

stator frame of reference as compared to case II.

Case I (b), with the 2D absorbing inlet boundary condition, is a little bit different from the other cases. This might have something to do with the modification of the cut off acoustic waves in the boundary condition, but on the other hand the boundary condition is not stable without the modification. In this specific case it is mode $m=-26$ that is troublesome. According to $2 \mathrm{D}$ characteristic theory it goes from being cut off to cut on inside the duct, and the decomposition into upstream and downstream traveling waves fails. On a small radial interval around the radial position where the transition from cut off to cut on is located, according to the 2D analysis, the decomposed Fourier coefficients of pressure for mode $m=-26$ each have a larger absolute value than the undecomposed Fourier coefficient, but the sum of the two decomposed complex numbers is equal to the undecomposed complex value. This is the reason for why the modification is necessary. Figure 9 shows the absolute value of the Fourier coefficient to mode $m=-26$ together with the absolute values of the $2 \mathrm{D}$ characteristic decomposition of it. It can be seen that the decomposed Fourier coefficients both have too large an absolute value.

Figure 10 show the propagating/cut on pressure modes at the inlet section. The largest difference is seen in $m=10$ where case I (a) stands for itself, but there are significant differences in the other modes as well. Case II (b) and (c) show good agreement as it should since it is exactly the same case solved with two different methods.

\section{VI.D. 3D mode analysis}

The solutions have been decomposed into radial modes and their characteristics by 3D analysis to better quantify the performance of the absorbing boundaries. ${ }^{19}$ The power level of the cut on modes with the same tangential mode number are summed up, and the difference in sound power between the modes that propagate towards the inlet boundary and away from the inlet boundary is calculated for each tangential mode number; see Table 2 . Note that $\Omega_{\text {rotor }}$ is negative and therefore $n$ becomes negative for positive frequencies.

An interesting fact is that almost all cut on pressure modes that propagate towards the inlet are better absorbed in case II (a) than case I (a). There is no buffer layer at the inlet in either of these two cases, and the only difference is that the specified rotor wake is stationary at the inlet in case II (a) whereas the wake rotates at the inlet in case I (a). Because of this, the $1 \mathrm{D}$ absorbing boundary condition performs better in 
Table 2. Reflection of Cut on Pressure Modes at the Inlet. The Values for the Different Cases in the Table Show a Difference in Power Level (Decibel) of the Sum of Radial Modes With Same Tangential Mode Number Propagating Towards the Inlet Boundary Compared to Reflected. A Negative Number Means Amplification at the Inlet Boundary. The Wave Numbers and Wave Lengths are Calculated by 2D Analysis Close to the Shroud.

\begin{tabular}{cllllll}
\hline $\mathrm{n}$ & -1 & -2 & -2 & -3 & -3 & -3 \\
$\mathrm{k}$ & 1 & 1 & 2 & 1 & 2 & 3 \\
\hline $\mathbf{m}$ & $\mathbf{1 0}$ & $\mathbf{- 8}$ & $\mathbf{2 0}$ & $\mathbf{- 2 6}$ & $\mathbf{2}$ & $\mathbf{3 0}$ \\
\hline Case I a & 7.43 & 5.08 & 9.05 & -0.36 & 11.2 & 9.96 \\
Case I b & 10.62 & 4.77 & 9.2 & 2.04 & $\mathbf{1 3 . 6 2}$ & 11.66 \\
Case II a & $\mathbf{1 3 . 4 6}$ & 6.75 & 15.7 & 2.18 & 10.08 & 15.35 \\
Case II b & 13.09 & $\mathbf{7 . 4 8}$ & $\mathbf{1 7 . 9 1}$ & 3.62 & 11.95 & $\mathbf{1 9 . 3 8}$ \\
Case II c & 12.77 & 7.33 & 16.17 & $\mathbf{3 . 7 4}$ & 13.28 & 16.96 \\
\hline \hline atan $\left(\frac{k_{\theta}}{k_{x}}\right)$ & $-20^{\circ}$ & $10^{\circ}$ & $-20^{\circ}$ & $26^{\circ}$ & $-1^{\circ}$ & $-20^{\circ}$ \\
$\frac{\lambda_{x}}{L_{B L}^{i n}}$ & 1.1 & 0.66 & 0.56 & 0.57 & 0.39 & 0.38 \\
\hline
\end{tabular}

the rotating frame of reference. If a buffer layer is applied at the inlet, as in case II (b,c), all modes except $m=10$ are better absorbed. Mode $m=10$ would probably be much better absorbed in case II (b,c) if the buffer layer is extended, since the axial wave length according to $2 \mathrm{D}$ analysis close to the shroud is larger than the axial length of the inlet buffer layer. ${ }^{4}$ This is not always possible though since the gap between the rotor and the stator is limited. It can be seen in Table 2 that the inlet buffer layer has almost no effect on mode $m=10$ when comparing case II $(\mathrm{a}, \mathrm{b}, \mathrm{c})$.

The 2D absorbing b.c., case I (b), performed better than the 1D absorbing b.c., case I (a), for all but one mode, $m=-8$, but compared to the cases with a rotating inlet section it was only better for mode $m=2$. The $2 \mathrm{D}$ absorbing b.c. takes care of the tangential variations and should therefore be better for modes with a high wave angle. This is probably true but as can be seen Table 2 it is not the only important factor. The radial variations, seen in Fig. 10, are of equal or more importance and an inlet buffer layer damp both kinds.

A more general observation is that modes that spin in the opposite direction to the rotor are absorbed better than modes that spin in the same direction. Another trend is that the higher the frequency in the rotor frame of reference (higher stator BPF number, $\mathrm{k}$ ) the better the modes are damped for case II. The reason for this might be that the high frequency modes are more dissipated in the rotating frame of reference and therefor better absorbed by the rotating inlet zone.

\section{Conclusions}

A method for how to apply an inlet buffer layer in nonlinear rotor wake/stator interaction computations has been developed and tested on a realistic stator vane geometry of a high bypass ratio fan stage. The inlet buffer layer is implemented as a low pass filter in a rotating inlet zone, where the rotor wake is stationary, and a chorochronic rotor-stator interface connects the rotating zone with the stationary stator domain.

The harmonic balance technique was also tested and good results were obtained for the test case with the rotating inlet buffer layer. Convergence was not obtained for a case without a rotating inlet section because of a slowly growing instability in the boundary layer at the hub between the inlet and the stator vane. The instability only appeared when the harmonic balance technique was used and it is possible that some modifications with the solver can solve this problem.

The results show that the 1D absorbing boundary condition absorbs a larger portion of an incoming oblique wave in the rotating frame of reference, without any inlet buffer layer, compared to the $1 \mathrm{D}$ absorbing b.c. in the stationary frame of reference. The 1D absorbing b.c. in the rotating frame of reference was also better than the $2 \mathrm{D}$ absorbing b.c. in a stationary frame of reference for five out of six analyzed cut on modes. The reason is that the specified rotor wake is stationary at the inlet in the rotating frame of reference and rotating at the inlet in the stationary frame of reference. Adding a buffer layer to the rotating inlet section can improve the absorption of approaching waves if the buffer layer is well tuned. 


\section{Acknowledgment}

Computer time at SNIC (Swedish National Infrastructure for Computing) resources at the National Supercomputer Center (NSC) in Linköping, Sweden, is gratefully acknowledged.

\section{References}

${ }^{1}$ Engquist, B. and Majda, A., "Absorbing Boundary Conditions for Numerical Simulation of Waves," Math. Comp., Vol. 31, No. 139, 1977, pp. 629-651.

${ }^{2}$ Hedstrom, G. W., "Nonreflecting Boundary Condition for Nonlinear Hyperbolic Systems," J. Comp. Phys., Vol. 30, 1979, pp. 222-237.

${ }^{3}$ Chassaing, J. C. and Gerolymos, G. A., "Time-Domain Implementation of Nonreflecting Boundary-Conditions for the Nonlinear Euler Equations," App. Math. Modelling, Vol. 31, 2007, pp. 2172-2188.

${ }^{4}$ Richards, S. K., Zhang, X., Chen, X. X., and Nelson, P. A., "The Evaluation of Non-Reflecting Boundary Conditions for Duct Acoustic Computation," J. Sound Vib., Vol. 270, 2004, pp. 539-557.

${ }^{5}$ Olausson, M. and Eriksson, L.-E., "An Absorbing Inlet Buffer Layer for Rotor Wake/Stator Time Domain Computations," No. GT2009-59346, ASME Turbo Expo 2009, Orlando, Florida, USA, 2009.

${ }^{6}$ Eriksson, L.-E., "Development and Validation of Highly Modular Flow Solver Versions in G2DFLOW and G3DFLOW," Internal report 9970-1162, Volvo Aero Corporation, Sweden, 1995.

${ }^{7}$ Andersson, N., A Study of Subsonic Turbulent Jets and Their Radiated Sound Using Large-Eddy Simulation, Ph.D. thesis, Division of Fluid Dynamics, Chalmers University of Technology, Gothenburg, 2005.

${ }^{8}$ Stridh, M., Modeling Unsteady Flow Effects in $3 D$ Throughflow Calculations, Ph.D. thesis, Department of Applied Mechanics, Division of Fluid Dynamics, Chalmers University of Technology, Gothenburg, 2006.

${ }^{9}$ Olausson, M., "Turbomachinery Aeroacoustic Calculations using a Time Domain Chorochronic Method," Licentiate Thesis, Division of Fluid Dynamics, Dept. of Applied Mechanics, Chalmers University of Technology, Gothenburg, 2008.

${ }^{10}$ Gerolymos, G. A., Michon, G. J., and Neubauer, J., "Analysis and Application of Chorochronic Periodicity in Turbomachinery Rotor/Stator Interaction Computations," J. Prop. and Power, Vol. 18, 2002, pp. 1139-1152.

${ }^{11}$ Schnell, R., Numerische Simulation des Akustischen Nahfeldes einer Triebwerksgebläsestufe, Ph.D. thesis, Fakultät V Verkehrs- und Maschinensysteme der Technischen Universität Berlin, 2004.

${ }^{12} \mathrm{Li}, \mathrm{H}$. D. and He, L., "Single-Passage Solution of Three-Dimensional Unsteady Flows in a Transonic Fan Rotor," Proceedings of the Institution of Mechanical Engineers, Part A: J. of Power and Energy, Vol. 215, 2001, pp. 653-662.

${ }^{13} \mathrm{Li}, \mathrm{H}$. D. and He, L., "Single-Passage Analysis of Unsteady Flows around Vibrating Blades of a Transonic Fan under Inlet Distortion," ASME J. of Turbomachinery, Vol. 124, 2002, pp. 285-292.

${ }^{14}$ Olausson, M., Eriksson, L.-E., and Baralon, S., "Nonlinear Rotor Wake/Stator Interaction Computations," No. 1307, The 18th ISABE meeting, Beijing, China, 2007.

${ }^{15}$ Olausson, M., Eriksson, L.-E., and Baralon, S., "Evaluation of Nonlinear Rotor Wake/Stator Interaction by using Time Domain Chorochronic Solver," No. 0064, The 8th ISAIF meeting, Lyon, France, 2007.

${ }^{16}$ Lebrun, M. and Favre, C., "Fan-OGV Unsteady Navier-Stokes Computation using an Adapted Acoustic Mesh," The 10th AIAA/CEAS Aeroacoustics Conference, Manchester, United Kingdom, 2004.

${ }^{17}$ Tyler, J. M. and Sofrin, T. G., "Axial Flow Compressor Noise Studies," SAE Transactions, Vol. 70, 1962, pp. 309-332.

${ }^{18}$ Baralon, S., Eriksson, L.-E., Billson, M., and Andersson, N., "Evaluation of Advanced Prediction Methods for Aero Engine Exhaust Noise," No. 1190, The 17th ISABE meeting, Munich, Germany, 2005.

${ }^{19}$ Verdon, J. M., "Linearized Unsteady Aerodynamic Analysis of the Acoustic Response to Wake/Blade-Row Interaction," NASA/CR-2001-210713, 2001.

${ }^{20}$ Billson, M., Computational techniques for turbulence generated noise, Ph.D. thesis, Division of Thermo and Fluid Dynamics, Chalmers University of Technology, Gothenburg, 2004.

${ }^{21}$ Hall, K. C., Thomas, J. P., and Clark, W. S., "Computation of Unsteady Nonlinear Flows in Cascades Using a Harmonic Balance Technique," AIAA journal, Vol. 40, 2002, pp. 879-886.

${ }^{22}$ Gopinath, A. K., van der Weide, E., Alonso, J. J., Jameson, A., Ekici, K., and Hall, K. C., "Three-Dimensional Unsteady Multi-stage Turbomachinery Simulations using the Harmonic Balance Technique," No. 892, The 45th AIAA Aerospace Sciences Meeting and Exhibit, Reno, Nevada, 2007. 

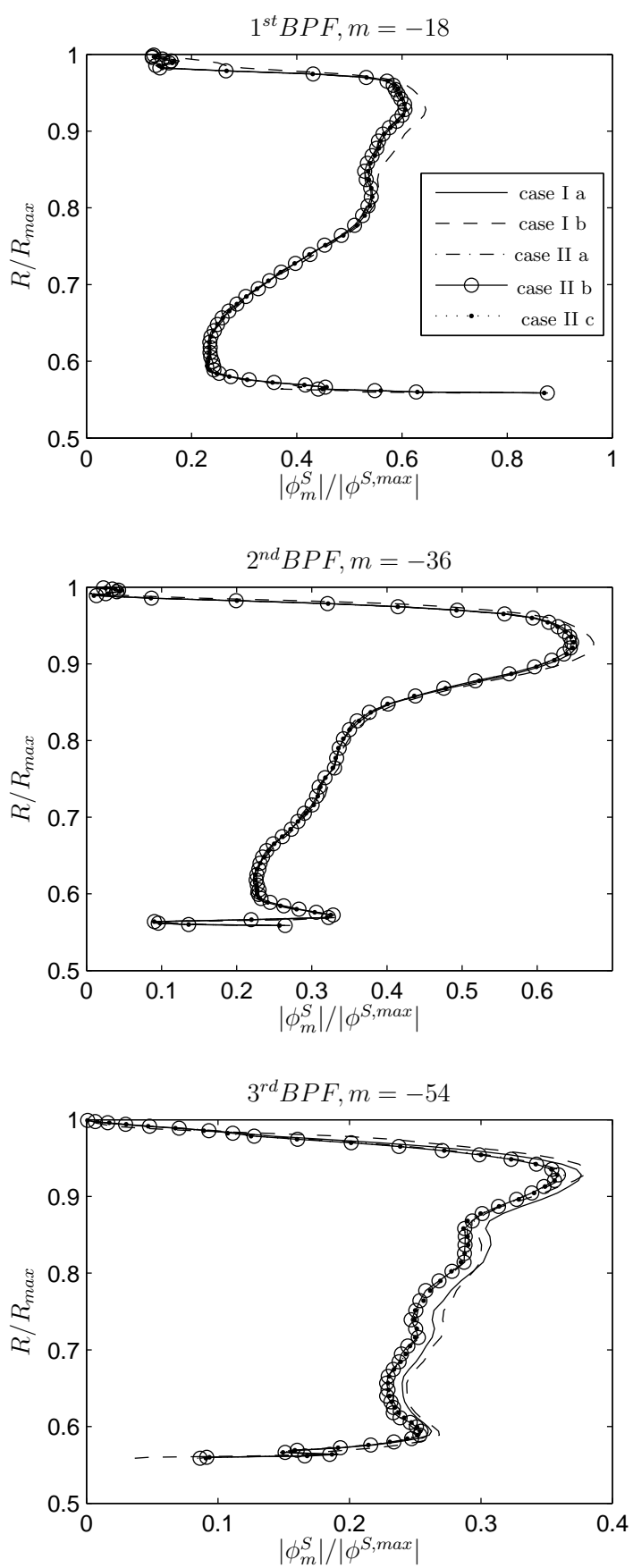

Figure 7. Entropy Modes Between Inlet and Stator

12 of 15

American Institute of Aeronautics and Astronautics 

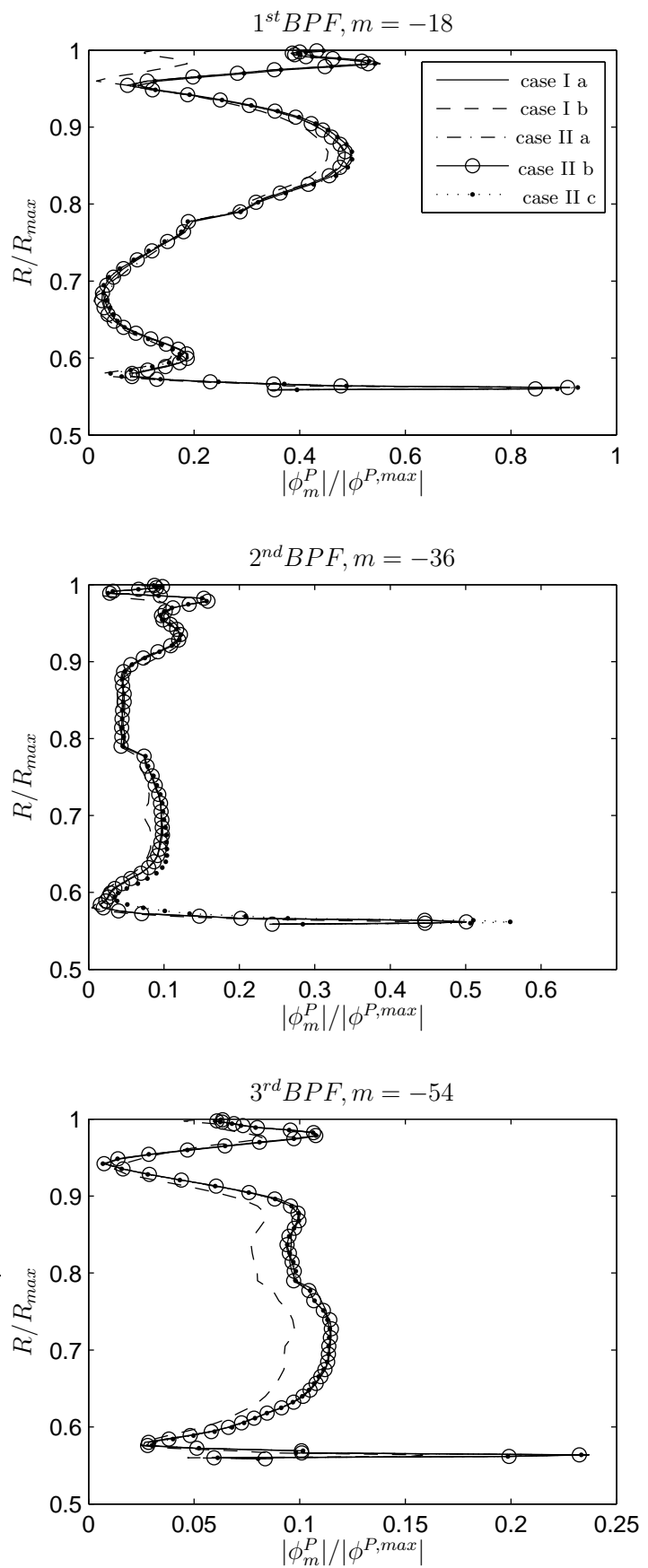

Figure 8. Rotor wake pressure Modes Between Inlet and Stator 


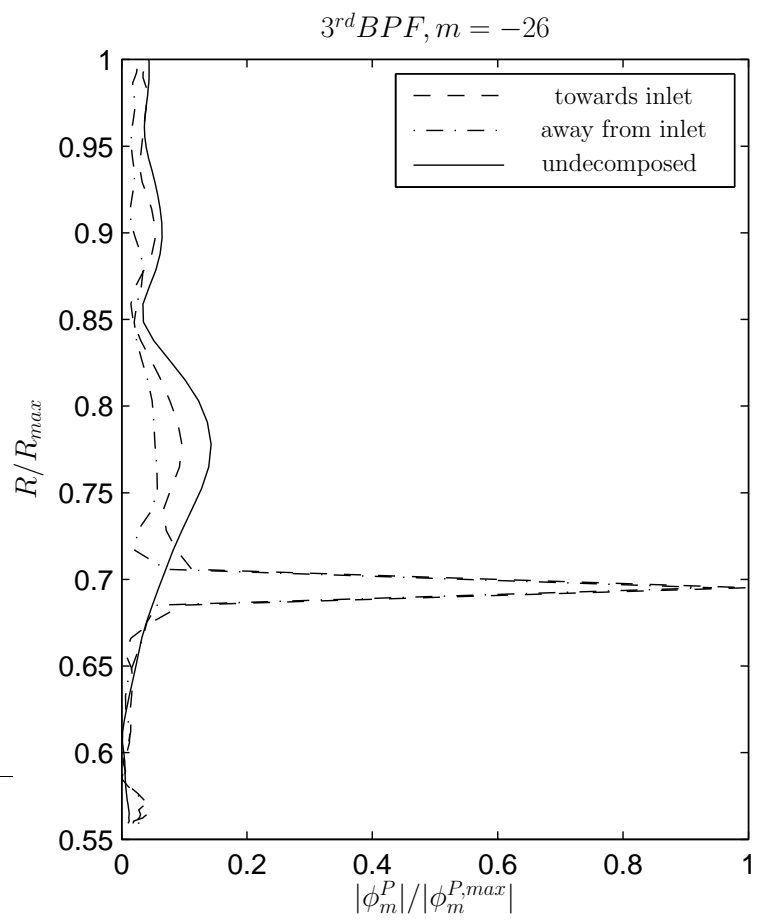

Figure 9. 2D Characteristic Decomposition of Pressure Mode $M=-26$ Between Inlet and Stator for Case I (a)

14 of 15

American Institute of Aeronautics and Astronautics 

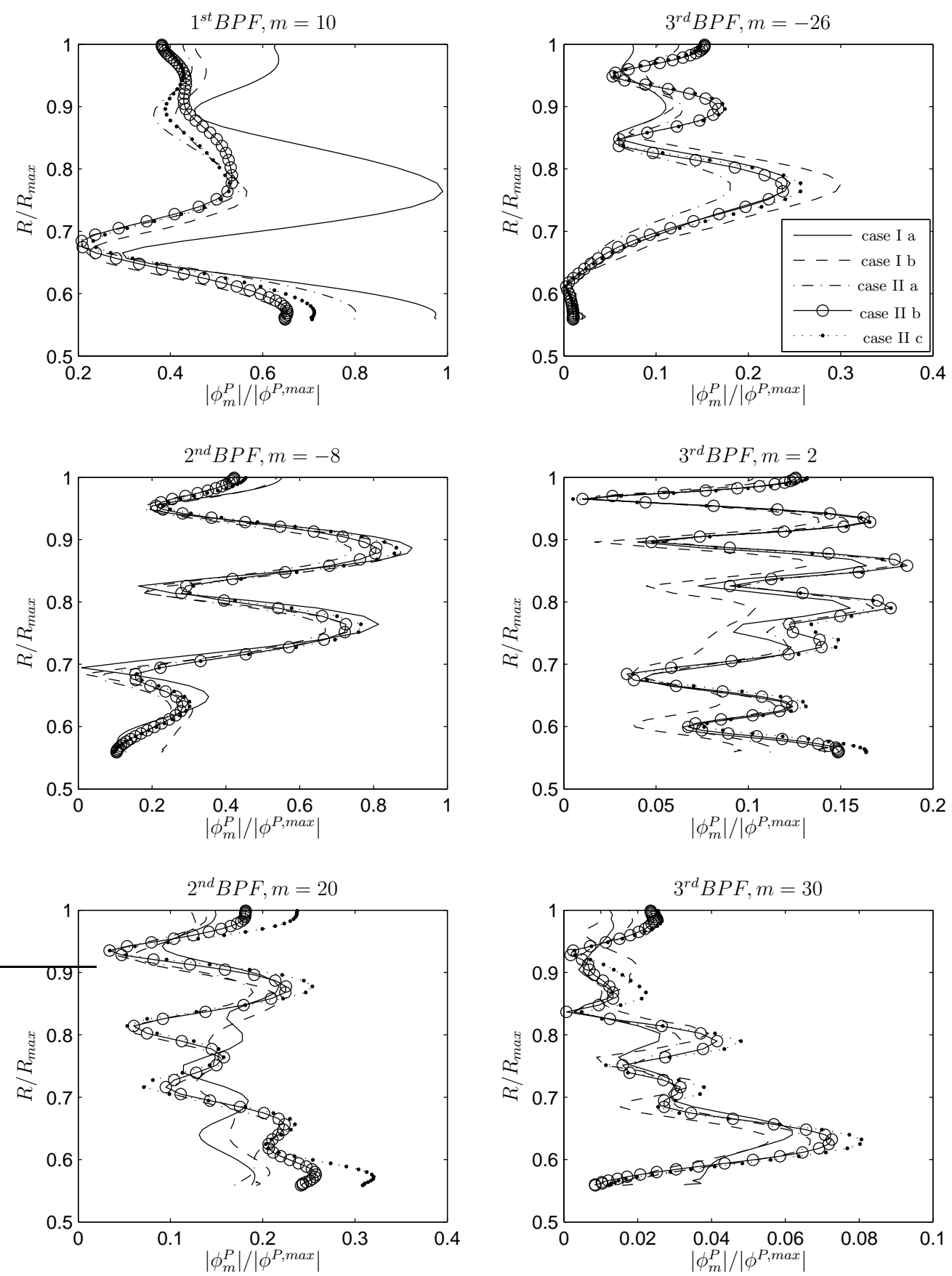

Figure 10. Cut-on pressure Modes Between Inlet and Stator

\section{5 of 15}

